\title{
The Efficacy of Sodium Bisulfate Salt (SBS) Alone and Combined With Peracetic Acid (PAA) as an Antimicrobial on Whole Chicken Drumsticks Artificially Inoculated With Salmonella Enteritidis
}

\author{
Dana K. Dittoe ${ }^{1}$, Julie A. Atchley ${ }^{1}$, Kristina M. Feye ${ }^{1}$, Jung Ae Lee ${ }^{2}$, Carl J. Knueven ${ }^{3}$ and \\ Steven C. Ricke ${ }^{1 *}$ \\ 1 Department of Food Science and Center for Food Safety, University of Arkansas, Fayetteville, AR, United States, \\ ${ }^{2}$ Agricultural Statistics Laboratory, University of Arkansas, Fayetteville, AR, United States, ${ }^{3}$ Jones-Hamilton Co., Walbridge, \\ $\mathrm{OH}$, United States
}

OPEN ACCESS

Edited by:

Ryan Arsenault,

University of Delaware, United States

Reviewed by:

Christine Elizabeth Ruth Dodd, University of Nottingham,

United Kingdom

Christian Visscher,

University of Veterinary Medicine

Hannover, Germany

*Correspondence:

Steven C. Ricke

sricke@uark.edu

Specialty section:

This article was submitted to Veterinary Infectious Diseases,

a section of the journal

Frontiers in Veterinary Science

Received: 01 November 2018 Accepted: 11 January 2019

Published: 30 January 2019

Citation:

Dittoe DK, Atchley JA, Feye KM Lee JA, Knueven CJ and Ricke SC

(2019) The Efficacy of Sodium

Bisulfate Salt (SBS) Alone and Combined With Peracetic Acid (PAA) as an Antimicrobial on Whole Chicken Drumsticks Artificially Inoculated With

Salmonella Enteritidis.

Front. Vet. Sci. 6:6.

doi: 10.3389/fvets.2019.00006
The presence of Salmonella spp. on poultry products is one of the leading causes of foodborne illness in the United States. Therefore, novel antimicrobial substances are being explored as potential interventions in poultry processing facilities. The objective of the current study was to evaluate the efficacy of varying concentrations of sodium bisulfate salt, SBS, alone or in combination with peracetic acid, PAA, in $15 \mathrm{~s}$ whole part dips. Ninety six drumsticks (4 replications, 8 treatments, 3 days) were inoculated separately in a $400 \mathrm{~mL}$ solution of nalidixic resistant (NA) Salmonella Enteritidis $\left(10^{7}\right.$ $\mathrm{CFU} / \mathrm{mL}$ ) and allowed to adhere for 60 to $90 \mathrm{~min}$ at $4^{\circ} \mathrm{C}$ for a final concentration of $10^{6}$ CFU/g. The experimental treatments included: a no treatment (control), and $15 \mathrm{~s}$ dips in $300 \mathrm{~mL}$ of tap water alone (TW) or with the addition of $1 ; 2$; and 3\% SBS; 1 ; 2 ; and $3 \%$ SBS+PAA. After treatment, drumsticks were stored at $4^{\circ} \mathrm{C}$ until microbial sampling was conducted. On d $0, \mathrm{I}$, and 3, drumsticks were rinsed in $150 \mathrm{~mL}$ of $\mathrm{nBPW}$ for $1 \mathrm{~min}, 100$ $\mu L$ of rinsate was serially diluted, spread plated on XLT4+NA (20 $\mu \mathrm{g} / \mathrm{mL})$, and incubated aerobically at $37^{\circ} \mathrm{C}$ for $24 \mathrm{~h}$. Log-transformed counts were analyzed using a randomized complete block design (day) using One-Way ANOVA, polynomial contrasts, and pairwise comparisons with means being separated by Tukey's HSD with a significance level of $P \leq 0.05$. A treatment by day interaction $(P=0.14071)$ was not substantial. Thus, the treatment effect was investigated separately by days. Over time, a linear trend was observed in S. Enteritidis concentration when SBS was increased $(1<2<3 \%)$. The concentration of $S$. Enteritidis was different between $1 \%$ SBS and $1 \%$ SBS+PAA on d 0. However, the level of $S$. Enteritidis was not different among drumsticks treated in 2 and $3 \%$ SBS and 2 and 3\% SBS+PAA across d 0, 1, 3. The application of 3\% SBS alone or in combination with 200 ppm of PAA is capable of reducing the presence of Salmonella over a 3-d refrigeration period; potentially increasing the safety of poultry products for consumers.

Keywords: S. Enteritidis, sodium bisulfate salt, processing, poultry, part dips 


\section{INTRODUCTION}

There is a need to enhance food safety strategies in the poultry industry. In the past 25 years, numerous steps have been taken to lessen the contamination of poultry products. The implantation of the Hazzard Analysis Critical Control Point (HACCP) in 1996 (1) and Food Safety Modernization Act (FSMA) in 2011 (2) established rules and guidelines for the food industry to follow in order to reduce the intensity and amount of foodborne illnesses. Although these strategies have had an impact on the incidence of foodborne illness, they are not entirely sufficient alone. As a result, the Center for Disease Control and Prevention (CDC) has set limits and goals on microbial reduction in the poultry industry. One pathogen the CDC is particularly interested in is Salmonella, especially Enteritidis, with outbreaks linked to poultry and eggs $(3,4)$. The CDC aims to lower Salmonella incidence by $5 \%$ by the year 2020 (5). In order to meet the CDC's goal, further intervention strategies must be integrated into the poultry industry.

In poultry processing facilities in the United States, one of the most commonly used methods to decontaminate poultry meat are antimicrobial washes and sprays at various locations during processing (6). Traditionally, chlorine and peracetic acid (PAA) are the antimicrobials of choice in the chiller, post chiller, spray cabinets, and part dips in poultry processing facilities (6). Recently, alternative antimicrobials have emerged for industrial application such as organic acids, phosphates, chlorine derivatives, and hydrogen peroxide solutions (7, 8). Antimicrobials that lower the $\mathrm{pH}$ of the surrounding environment are promising; however, Gram-negative species, such as Salmonella, are capable of developing resistance to organic acids due to the presence of LPS (9). In addition, bacteria such as Salmonella can build a tolerance to stressful environments (10).

Inorganic acids also have the potential to induce the resistance of pathogens, such as Salmonella, to a low environmental $\mathrm{pH}$ (11). Research conducted by Foster and Hall (11) revealed that in order for inorganic acids to induce an acid tolerance response new protein synthesis and the development of a $\mathrm{pH}$ homeostasis system is required. As a consequence, both the acid tolerance response and acid-shock proteins are required for Salmonella to survive acidic conditions induced by inorganic acids (1214). Sodium bisulfate (SBS), an inorganic acid, has demonstrated the ability to decrease the extracellular $\mathrm{pH}$ to around 2 with a $\mathrm{pK}_{\mathrm{a}}$ of 1.9 (15). If there is a mild decrease in the extracellular $\mathrm{pH}$, Salmonella reduce cytoplasmic $\mathrm{pH}$ to maintain a neutral state (16). This response is incredibly arduous for Salmonella and can lead to cell death (16). Because of these features, SBS demonstrates the potential to be a valid antimicrobial over other organic and inorganic acids as it has the ability to create a highly acidic environment that is not easily adapted to.

Historically, SBS has been utilized commercially as an acidifier on poultry litter. When used as a litter amendment at high doses, SBS not only reduced ammonia volatilization from poultry litter but also the presence of Salmonella (17). Additionally, the dietary inclusion of SBS decreased the shedding of Salmonella into the litter (18). Pertinent to poultry processing, SBS was used as an antimicrobial rinse agent on apples to reduce artificially inoculated Listeria monocytogenes (19). The Environmental Protection Agency has declared SBS as a safer choice as an antimicrobial and processing aid (20). In addition, according to the World Health Organization (WHO), the use of SBS is approved with no restrictions on allowable daily intake in over 150 countries that recognize the WHO codex (21). Because of all of the preliminary data as well as the Environmental Protection Agency designation, it is evident that SBS could be a valid agent for reducing Salmonella, during multiple stages of poultry production, including processing. Therefore, it was the objective of the current study to investigate the potential of SBS as an antimicrobial intervention in poultry processing by determining the efficacy of SBS alone (1,2, and 3\%) or in combination with PAA (200 ppm) on mitigating the presence of a nalidixic acid (NA) resistant strains of Salmonella Enteritidis on whole chicken drumsticks.

\section{MATERIALS AND METHODS}

\section{Whole Chicken Drumsticks and Salmonella Screening}

A total of 96 drumsticks (8 treatments, 3 days, 4 replicates) were obtained from a local supermarket no longer than $24 \mathrm{~h}$ before the onset of the study and chosen based on the furthest expiration date. Prior to the start of the study, one drumstick was screened for the background unintended presence of Salmonella. One drumstick was rinsed in $150 \mathrm{~mL}$ of neutralizing Buffered Peptone Water [nBPW; (22)] and manually agitated for $1 \mathrm{~min}$. Subsequently, $100 \mu \mathrm{L}$ of the rinsate was spread plated onto Xylose Lysine Tergitol 4 with the addition of $20 \mu \mathrm{g} / \mathrm{mL}$ of NA (XLT $4+$ NA) and incubated for $24 \mathrm{~h}$ at $37^{\circ} \mathrm{C}$.

\section{Inocula Preparation}

Before the onset of the current study, a frozen stock of Salmonella Enteritidis that was selected to be resistant to $20 \mu \mathrm{g} / \mathrm{mL}$ of NA was streaked for isolation on Tryptic Soy Agar (TSA) and incubated for $24 \mathrm{~h}$ aerobically at $37^{\circ} \mathrm{C}$. Subsequently, one isolated colony from the incubated plate was streaked onto fresh TSA with the addition of $20 \mu \mathrm{g} / \mathrm{mL}$ of NA (TSA + NA) and incubated under the previously mentioned conditions. Simultaneously, an isolated colony was streaked onto XLT 4 plus $20 \mu \mathrm{g} / \mathrm{mL}$ of NA (XLT $4+$ NA) for confirmation and incubated aerobically at $37^{\circ} \mathrm{C}$ for $24 \mathrm{~h}$. An isolated colony from the incubated TSA + NA plate was then transferred to $40 \mathrm{~mL}$ of fresh Tryptic Soy Broth the addition of $20 \mu \mathrm{g} / \mathrm{mL}$ of NA (TSB +NA) and incubated aerobically at $37^{\circ} \mathrm{C}$ in a shaking incubator at $200 \mathrm{rpm}$ for 12 to $16 \mathrm{~h}$. The resulting cultures of $S$. Enteritidis were determined to contain $10^{8} \mathrm{CFU} / \mathrm{mL}$.

Directly following the overnight (12 to $16 \mathrm{~h}$ ) incubation of the $S$. Enteritidis cultures, the cultures were spun down at $18,000 \mathrm{~g}$ for $5 \mathrm{~min}$, decanted, followed by washing twice in $1 \times$ Phosphate Buffered Saline (PBS). After the final wash, the pellet was resuspended in $400 \mathrm{~mL}$ of sterile DI water. 


\section{Inoculation}

A separate inoculum of $S$. Enteritidis was utilized per replication of drumsticks (4 replications). Approximately 24 drumsticks were placed into sterile Whirl-Pak ${ }^{\circledR}$ (Nasco, Atkins, WI, USA) bags, where the inoculum was administered. The inoculated drumsticks were then massaged manually for $5 \mathrm{~min}$ and allotted 60 to $90 \mathrm{~min}$ at $4^{\circ} \mathrm{C}$ to allow for attachment.

\section{Treatment pH and Application}

Following inoculation, the eight experimental antimicrobial treatments were created by combining TW with the appropriate amounts of SBS and 200 ppm of PAA (Sigma-Aldrich, St. Louis, MO, USA) to create proper concentration of the following treatments: a no treatment (control), a $15 \mathrm{~s}$ dip in $300 \mathrm{~mL}$ of tap water alone (TW), and TW with the addition of 1\% SBS (TW+SBS1); 2\% SBS (TW+SBS2); 3\% SBS (TW+SBS3); 1\% SBS + PAA (TW+SBS1+PAA); $2 \%$ SBS + PAA (TW+SBS2+PAA); and $3 \%$ SBS + PAA (TW+SBS3+PAA). Before drumsticks were treated, one replicate of each treatment was analyzed for $\mathrm{pH}$ with a SympHony $\mathrm{pH}$ meter and probe (VWR International, Radnor, PA, USA). Immediately following the attachment period, the drumstick weights were recorded and the treatments were administered. The whole chicken drumsticks were independently dipped for $15 \mathrm{~s}$ into sterile Whirl-Pak bags containing the eight previously described treatments.

\section{Microbial Analysis and Salmonella Enumeration}

Following the $15 \mathrm{~s}$ dips, the drumsticks were transferred to new sterile Whirl-Pak bags and allowed to rest for $2 \mathrm{~min}$. The drumsticks were evaluated immediately on $\mathrm{d} 0$ or maintained at $4^{\circ} \mathrm{C}$ for an additional $24 \mathrm{~h}(\mathrm{~d} 1)$ or $72 \mathrm{~h}(\mathrm{~d} 3)$ and then analyzed for Salmonella Enteritidis concentration. At each time point posttreatment, $\mathrm{d} 0,1$, and 3, the drumsticks were rinsed with $150 \mathrm{~mL}$ of sterile nBPW. The Whirl-Pak bags containing the $150 \mathrm{~mL}$ of $\mathrm{nBPW}$ and drumsticks were then manually agitated for $1 \mathrm{~min}$ and the resulting rinsates were collected for downstream analysis.

Whole chicken drumstick rinsates were aliquoted to $1.5 \mathrm{~mL}$ microcentrifuge tubes and subsequently serially diluted to $10^{-6}$ (1:10 dilution factor). After diluting the samples, a $100 \mu \mathrm{L}$ aliquot of each dilution was spread plated onto XLT $4+$ NA $(20 \mu \mathrm{g} / \mathrm{mL})$ agar in duplicate using sterile spreaders. The plates were then inverted and incubated aerobically for $24 \mathrm{~h}$ at $37^{\circ} \mathrm{C}$. Only the plated dilutions with CFU counts between 30 and 300 were enumerated and recorded.

The following equation was utilized to calculate the CFU of Salmonella per gram of whole chicken drumstick:

$$
\frac{\left(\frac{\text { Number of colonies }}{0.1 m L \text { plated }}\right) * \text { Dilution Factor }}{\frac{\text { Drumstick Weight }(\mathrm{g})}{\text { Original Homogenate }(\mathrm{mL})}}
$$

\section{Statistical Analysis}

Each drumstick was randomly assigned to a treatment and a time point prior to analyses. The CFU of Salmonella were $\log _{10}$ transformed and reported on a $\log \mathrm{CFU}$ of $S$. Enteritidis per

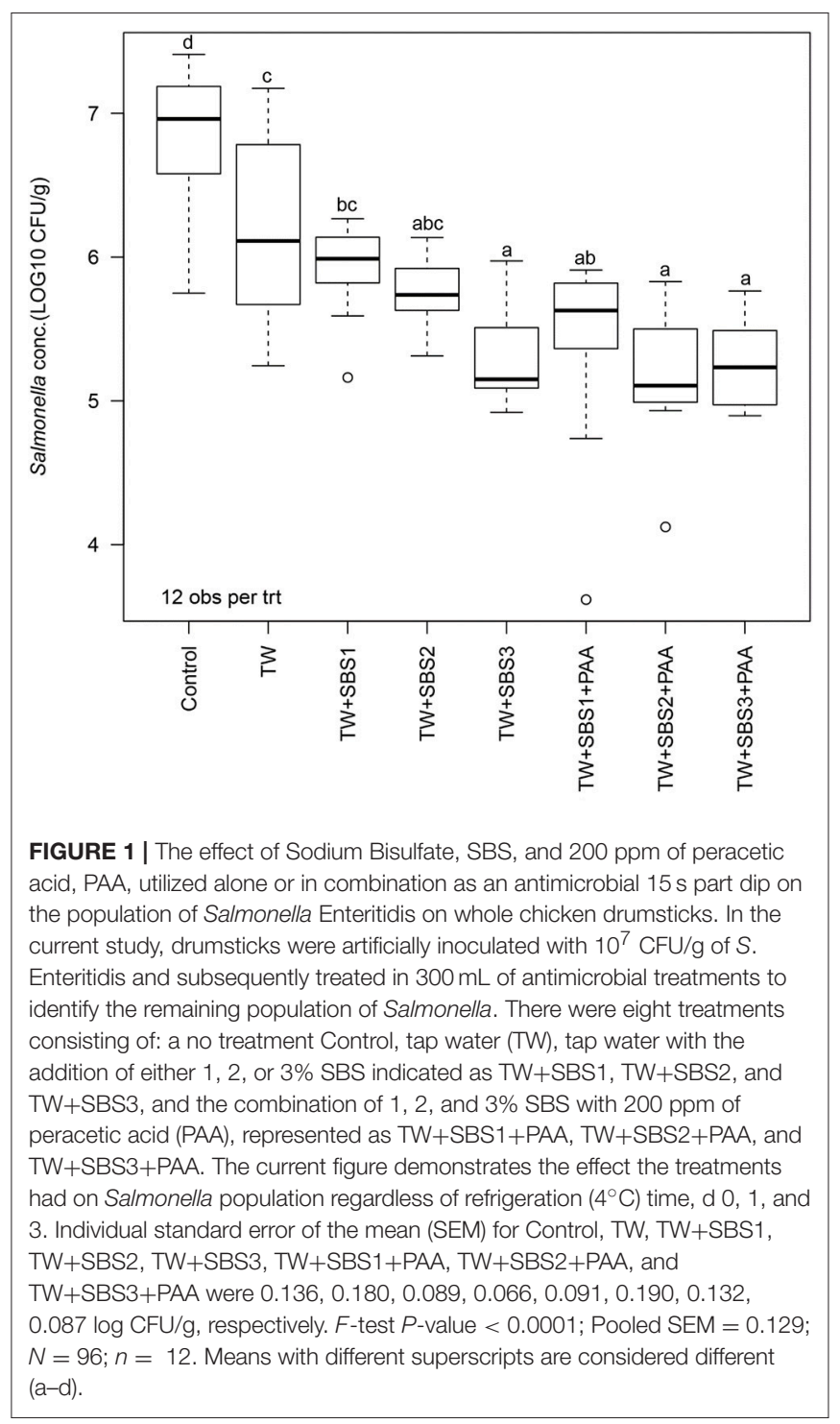

gram of drumstick basis (log CFU/g). The data were analyzed as a Randomized Complete Block design with replications $(n=4)$ where the blocks are designated as day, d 0, 1, and 3, using oneway ANOVA, polynomial contrasts, and pairwise comparisons. The differences were assessed statistically by using Tukey's protected HSD at 0.05 level of significance. Data analyses were performed in $\mathrm{R}$ version 3.3.2 (23).

\section{RESULTS}

In the current study, the overall one-way ANOVA did not produce a significant interaction between the block (day) and treatment $(P=0.1407)$. There was no main effect of day $(P=0.0948)$; however, there was a main effect of treatment $(P<0.0001$; Figure 1). Overall, all treatments, TW, TW+SBS1, TW+SBS2, TW+SBS3, TW+SBS1+PAA, TW+SBS2+PAA, and TW+SBS3+PAA $(6.22,5.93,5.76,5.28,5.42,5.19$, $5.27 \log \mathrm{CFU} / \mathrm{g})$, reduced the population of $S$. Enteritidis 


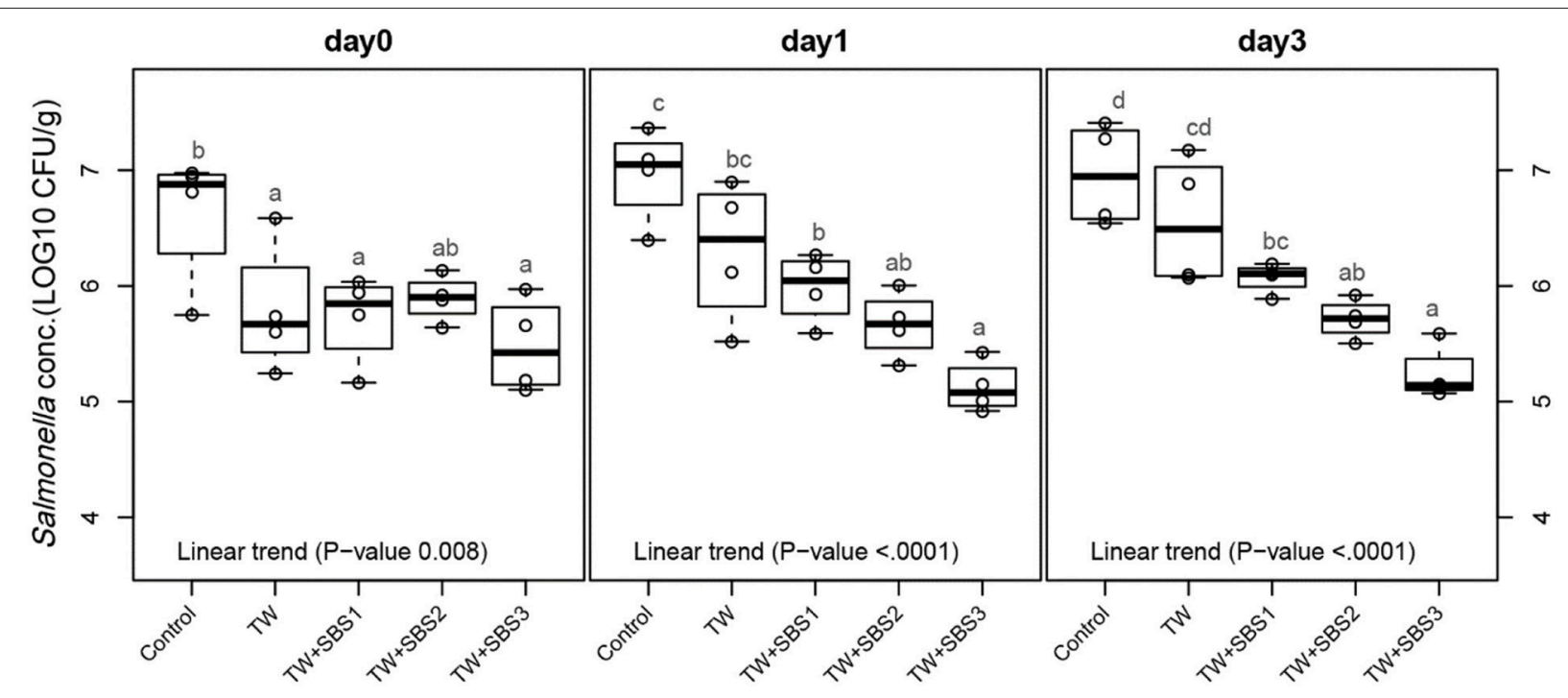

FIGURE 2 | The linear effect of Sodium Bisulfate, SBS, as an antimicrobial $15 \mathrm{~s}$ part dip on suppressing the population of Salmonella Enteritidis on whole chicken drumsticks on d 0,1 , and 3 . Drumsticks were artificially inoculated with $10^{7} \mathrm{CFU} / \mathrm{g}$ of $\mathrm{S}$. Enteritidis and subsequently treated in $300 \mathrm{~mL}$ of antimicrobial treatments to identify the remaining population of Salmonella. There were eight antimicrobial treatments consisting of: a no treatment Control, tap water (TW), tap water with the addition of either 1,2 , or $3 \%$ SBS indicated as TW+SBS1, TW+SBS2, and TW+SBS3, and the combination of 1,2 , and $3 \%$ SBS with 200 ppm of peracetic acid (PAA), represented as TW+SBS1+PAA, TW+SBS2+PAA, and TW+SBS3+PAA. In the current figure, a linear trend was investigated for the incremental increase in SBS concentration, Control $(n=4)$, TW $(n=4)$, TW+SBS1 (TW+SBS1 and TW+SBS1+PAA, $n=8)$, TW+SBS2 (TW+SBS2 and TW+SBS2+PAA, $n=8)$, and TW+SBS3 (TW+SBS3 and TW+SBS3+PAA, $n=8$ ), over a 3-d refrigeration period at $4^{\circ} \mathrm{C}$. Individual SEM for Control, TW, TW+SBS1, TW+SBS2, and TW+SBS3 was 0.292, 0.284, 0.281, 0.229, 0.146 log CFU/g for d 0, 0.205, 0.309, 0.092, 0.122, and 0.089 log CFU/g for d 1, and 0.223, 0.278, 0.082, 0.131, and 0.067 for d 3 , respectively. Pooled SEM for d 0 is $0.227,0.196$ for $d 1$, and 0.175 for $\mathrm{d} 3 ; N=32$. Means with different superscripts are considered different (a-d).

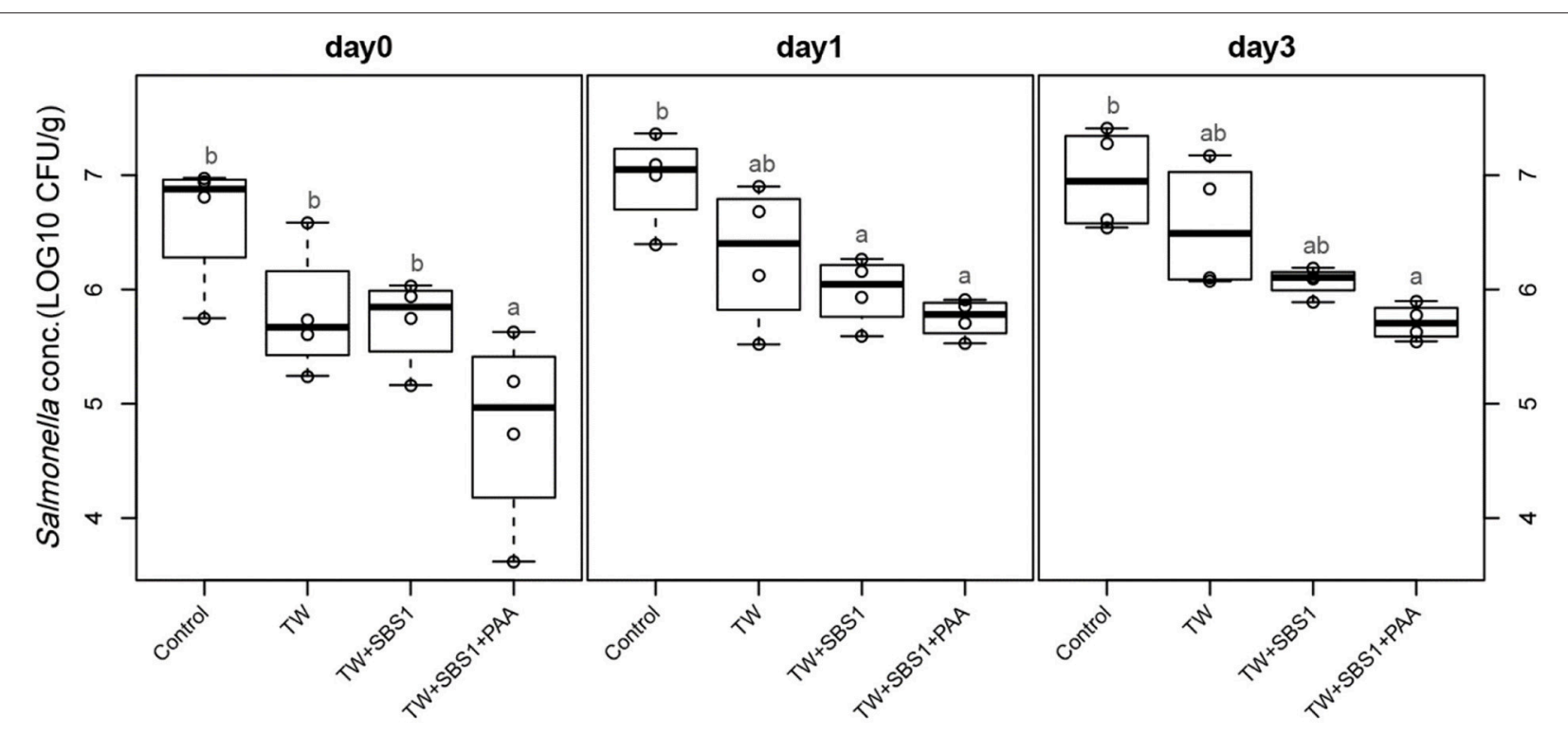

FIGURE 3 | The comparative effect of $1 \%$ Sodium Bisulfate, SBS, and 200 ppm of peracetic acid, PAA, utilized alone or in combination as antimicrobial 15 s part dips on the presence of Salmonella Enteritidis on whole chicken drumsticks on d 0, 1, and 3 . In the current study, drumsticks were artificially inoculated with $10^{7}$ CFU/g of S. Enteritidis and subsequently treated in $300 \mathrm{~mL}$ of antimicrobial treatments to identify the remaining population of Salmonella. In the study, there were eight treatments, consisting of: a no treatment Control, tap water (TW), tap water with the addition of either 1, 2, or 3\% SBS indicated as TW+SBS1, TW+SBS2, and TW+SBS3, and the combination of 1, 2, and 3\% SBS with 200 ppm of peracetic acid (PAA), represented as TW+SBS1+PAA, TW+SBS2+PAA, and TW+SBS3+PAA. However, in the current figure only the Control, TW, TW+SBS1, and TW+SBS1+PAA is represented and is separated by d 0 , 1 , and 3 of $4{ }^{\circ} \mathrm{C}$ incubation. Individual SEM for Control, TW, TW+SBS1, and TW+SBS1+PAA was 0.293, 0.284, 0.196, and 0.432 for d 0; 0.205, 0.309, 0.150, and 0.086 for d 1; and $0.223,0.278,0.064$, and 0.078 for $\mathrm{d} 3$, respectively. P-value for $\mathrm{d} 0$ is $0.0116,0.0067$ for $\mathrm{d} 1$, and 0.0024 for $\mathrm{d} 3$; Pooled SEM for $\mathrm{d} 0$ is 0.313 , 0.204 for $\mathrm{d} 1$, and 0.0024 for d 3 ; Per day $N=16$ and $n=4$. Means with different superscripts are considered different $(a, b)$. 


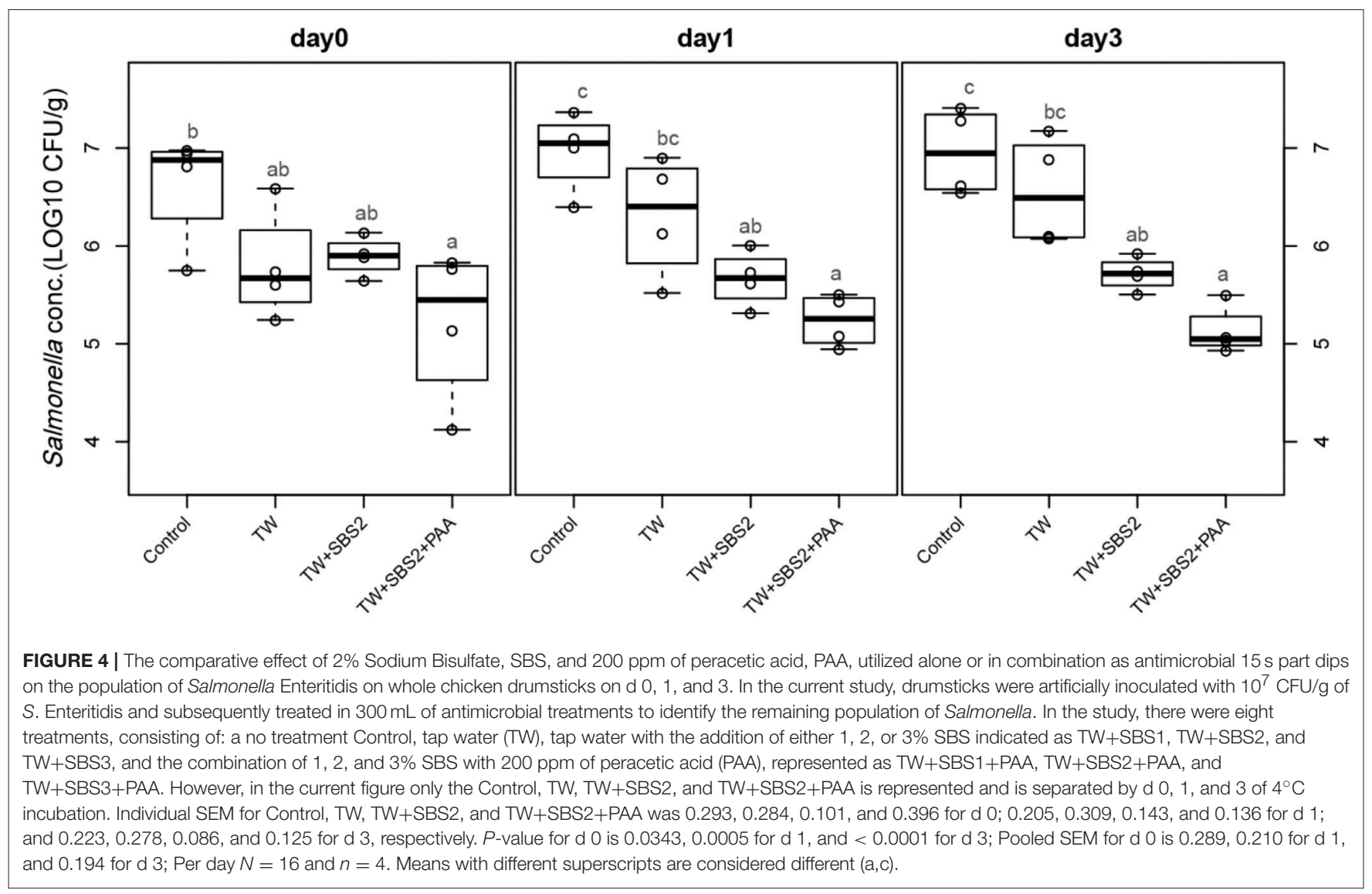

on the drumsticks compared to the no treatment control (6.85 log CFU/g). Also, drumsticks treated with TW+SBS3, TW+SBS2+PAA, and TW+SBS3+PAA (5.28, 5.19, and $5.27 \mathrm{log}$ CFU/g) had populations of $S$. Enteritidis 1 to $2 \log$ CFU per $g$ of drumstick lower than those treated with the Control, TW, and TW+SBS1 $(6.85,6.22$, and $5.93 \log \mathrm{CFU} / \mathrm{g})$.

Although there was not a significant interaction between treatment and day $(P=0.1407)$, the main effects of each treatment on each day were evaluated using polynomial contrasts and pairwise comparisons of treatments. Linear trends were investigated for the increasing concentrations of SBS within treatments: Control, TW, TW+SBS1 (SBS1 and SBS1+PAA), TW+SBS2 (SBS2 and SBS2+PAA), and TW+SBS3 (SBS3 and SBS3+PAA). By combining treatments with similar concentrations of SBS, negative linear trends of log CFU of Salmonella per gram of drumstick occurred as SBS increased $(1 \%<2 \%<3 \%)$ on $\mathrm{d} 0,1$, and $3(P=0.0008, P<0.0001$, $P<0.0001$, Figure 2), where $\mathrm{d} 1$ and 3 had distinct linear trends. On $\mathrm{d} 0$, there was no detectable difference between TW+SBS 1 , 2, and 3; however, on both $\mathrm{d} 1$ and 3, TW+SBS1 and TW+SBS3 had detectable differences with SBS3 yielding a lower population of $S$. Enteritidis per gram of drumstick (5.99 and $5.13 \mathrm{log}$ CFU/g on $\mathrm{d} 1$ and 6.07 and $5.24 \log \mathrm{CFU} / \mathrm{g}$ on d 3).

To further evaluate the effects of the treatments, TW+SBS and TW+SBS+PAA treatments were examined separately alongside the control and TW in pairwise comparisons by day (Figures 35). Although not statistically different, TW+SBS1+PAA and TW+SBS2+PAA treatments exhibited a lower presence of $S$. Enteritidis per gram of drumstick than those treated with TW+SBS1 and TW+SBS2 alone. Only on d 0 did TW+SBS1+PAA have a significantly lower population of $S$. Enteritidis than TW+SBS1 (4.80 and $5.72 \log$ CFU/g). Thus, both TW+SBS1+PAA and TW+SBS2+PAA treatments show a slight advantage over TW+SBS treatments. Drumsticks treated with TW+SBS3 and TW+SBS3+PAA did not yield the previously mentioned pattern. In fact, TW+SBS3+PAA (5.33, 5.28, and 5.19 $\log \mathrm{CFU} / \mathrm{g}$ on $\mathrm{d} 0,1$, and 3 , respectively) was not more effective at reducing the population of $S$. Enteritidis on drumsticks than TW+SBS3 (5.48, 5.13, and 5.24 log CFU/g on d 0, 1, and 3, respectively), primarily as time continued.

Though the $\mathrm{pH}$ of the treatments was not statistically analyzed, due to insufficient replication, there was a clear numerical difference between TW and treatments (Table 1). The mean $\mathrm{pH}$ of the TW solution was 7.42; whereas, the mean $\mathrm{pH}$ of $\mathrm{TW}+\mathrm{SBS} 1, \mathrm{TW}+\mathrm{SBS} 2, \mathrm{TW}+\mathrm{SBS} 3, \mathrm{TW}+\mathrm{SBS} 1+\mathrm{PAA}$, $\mathrm{TW}+\mathrm{SBS} 2+\mathrm{PAA}$, and TW+SBS3+PAA was $1.64,1.45,1.31$, $1.51,1.33$, and 1.29 , respectively. It should be noted that there was a numerical drop in the $\mathrm{pH}$ level of the TW+SBS1 and TW+SBS2 solutions when PAA was added. There was not a substantial drop in TW+SBS3 when PAA was added. 


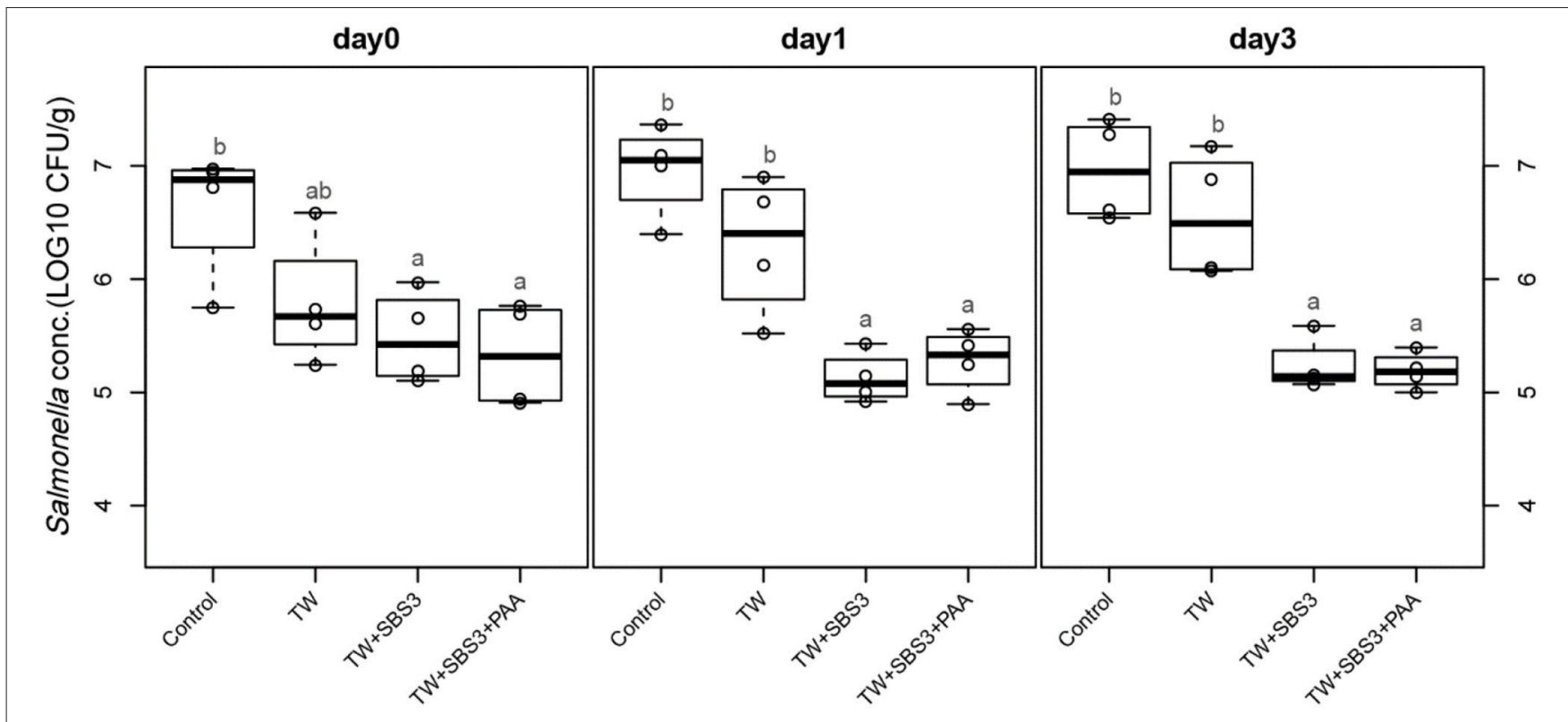

FIGURE 5 | The comparative effect of 3\% Sodium Bisulfate, SBS, and 200 ppm of peracetic acid, PAA, utilized alone or in combination as antimicrobial 15 s part dips on the presence of Salmonella Enteritidis on whole chicken drumsticks on d 0, 1, and 3. In the current study, drumsticks were artificially inoculated with $10^{7}$ CFU/g of $S$. Enteritidis and subsequently treated in $300 \mathrm{~mL}$ of antimicrobial treatments to identify the remaining population of Salmonella. In the study, there were eight treatments, consisting of: a no treatment Control, tap water (TW), tap water with the addition of either 1, 2, or 3\% sodium bisulfate (SBS) indicated as TW+SBS1, TW+SBS2, and TW+SBS3, and the combination of 1, 2, and 3\% SBS with 200 ppm of peracetic acid (PAA), represented as TW+SBS1+PAA, TW+SBS2+PAA, and TW+SBS3+PAA. However, in the current figure only the Control, TW, TW+SBS3, and TW+SBS3+PAA is represented and is separated by $\mathrm{d} 0$, 1 , and 3 of $4{ }^{\circ} \mathrm{C}$ incubation. Individual SEM for Control, TW, TW+SBS3, and TW+SBS3+PAA was 0.293, 0.284, 0.205, and 0.232 for d 0; 0.205, 0.309, 0.112, and 0.143 for d 1; and $0.223,0.278,0.119$, and 0.083 for $\mathrm{d} 3$, respectively. F-test $P$-values are 0.0169 for $\mathrm{d} 0,0.0001$ for $\mathrm{d} 1$, and < 0.0001 for $\mathrm{d} 3$; Pooled SEMs are 0.256 for $\mathrm{d} 0$, 0.206 for $d$, and 0.192 for $d 3$; Per day $N=16$ and $n=4$. Means with different superscripts are considered different (a,b).

\section{DISCUSSION}

Although the authors did not evaluate the Salmonella recovered in this study for invasion or infectivity, data presented herein is promising as it demonstrates the possibility of SBS to improve food safety. Throughout the course of this study, SBS treatments reduced the concentration of $S$. Enteritidis below the typical infectious dose of ingested Salmonella to humans, $10^{6}$ to $10^{8}$ CFU, though the infectious dose of Salmonella can vary based on the matrix and the immune status of the affected individual (24). Despite the fact that the infectious dose has been reported to be much lower in other studies (25), the log reduction of $1.75 \mathrm{CFU} / \mathrm{g}$ on $\mathrm{d} 3$ in the current study demonstrates the ability of SBS to effectively reduce pathogens to a potentially non-infectious dose for those who are not immunocompromised. Therefore, data presented herein warrants further investigations into whether or not treating poultry carcasses with SBS reduces salmonellosis.

The use of PAA has been shown to be an effective antimicrobial in poultry processing and its potential synergism with SBS (25). Two commercial acidifiers, acetic acid and hydrogen peroxide, are individually effective against pathogens. In combining both acids, synergism is demonstrated and yields PAA (26). This effect is likely driven by the acidification of the hydrogen peroxide by acetic acid (26). It is possible that the acidification of PAA may enhance its antimicrobial properties. As SBS is a strong acidifier with a $\mathrm{pK}_{\mathrm{a}}$ of 1.9 (15), it was important to evaluate the potential synergism between SBS and PAA. As demonstrated by the current study, the combination of $3 \%$ SBS and $200 \mathrm{ppm}$ of PAA had a lower $\mathrm{pH}$ (1.29) and reduced $S$. Enteritidis more than $1.7 \log$ CFU of $S$. Enteritidis per $g$ of drumstick. Consequently, the combination of SBS with PAA demonstrated similar trends in reducing Salmonella as other studies investigating the use of PAA alone. The application of $85 \mathrm{ppm}$ of PAA in a chilling tank resulted in a 1 log reduction (91.8\% reduction) of Salmonella-positive carcasses (27). In other research investigating the effects of PAA as a post-chill dip (10 or $20 \mathrm{~s}$ ), Nagel et al. (28) reported a 2-log reduction of $S$. Typhimurium among whole carcasses treated in a $20 \mathrm{~s}$ postchiller dip of 400 or 1000 ppm of PAA. Though the current study indicates the potential combinatorial effect of SBS and PAA, it also confirms the validity of SBS as an antimicrobial when used alone.

In previous studies, the use of SBS alone has demonstrated to be an effective antimicrobial agent. Previously, when SBS was applied as a pre-chill $90 \mathrm{~s}$ spray, it resulted in a $2.4 \mathrm{log}$ CFU reduction of $S$. Typhimurium (29). Another study similar to the one conducted herein demonstrated a similar reduction of Salmonella was exhibited in research by Yang et al. (30). Yang et al. (30) showed that a $17 \mathrm{~s}$ application of 5\% SBS in an inside-outside bird wash reduced Salmonella by $1.66 \log$ CFU per carcass. As demonstrated in the current and past studies, the acidification of water induced by SBS has the potential to 
TABLE 1 | The mean pH values of Sodium Bisulfate (SBS) salt and PAA, used alone or in combination when utilized as $15 \mathrm{~s}$ dip solutions ${ }^{\mathrm{a}}$.

\begin{tabular}{lc}
\hline Treatment $^{\mathbf{b}}$ & Mean $\mathbf{~ p H ~ o f ~ P a r t ~ D i p s ~}^{\mathbf{c}}$ \\
\hline Control & - \\
$\mathrm{TW}$ & 7.42 \\
$\mathrm{TW}+\mathrm{SBS} 1$ & 1.64 \\
$\mathrm{TW}+\mathrm{SBS} 2$ & 1.45 \\
$\mathrm{TW}+\mathrm{SBS3}$ & 1.31 \\
$\mathrm{TW}+\mathrm{SBS} 1+\mathrm{PAA}^{\mathrm{d}, \mathrm{e}}$ & 1.51 \\
$\mathrm{TW}+\mathrm{SBS} 2+\mathrm{PAA}^{\mathrm{d}, \mathrm{e}}$ & 1.33 \\
$\mathrm{TW}+\mathrm{SBS3}+\mathrm{PAA}^{\mathrm{d}, \mathrm{e}}$ & 1.29 \\
\hline
\end{tabular}

${ }^{a}$ Contact time: $15 \mathrm{~s}$ based on Morris recommendation and current industry practice.

${ }^{b}$ There were eight antimicrobial treatments consisting of: a no treatment Control, tap water (TW), tap water with the addition of either 1,2, or 3\% sodium bisulfate (SBS) indicated as $T W+S B S 1, T W+S B S 2$, and TW+SBS3, and the combination of 1, 2, and $3 \%$ SBS with 200 ppm of peracetic acid (PAA), represented as TW+SBS1+PAA, TW+SBS2+PAA, and $T W+S B S 3+P A A$.

${ }^{c}$ Mean $\mathrm{pH}$ of part dips was determined using a SympHony pH meter (WWR International, Radnor, PA). The mean $\mathrm{pH}$ of the solutions prepared for drumsticks was based on the four replicated experiments. There was no $\mathrm{pH}$ for the no treatment group (Control) as no solution was prepared.

d200 ppm concentration of PAA

e Peracetic acid solution; 39\% PAA; Sigma-Aldrich, 3050 Spruce St., St. Louis, MO.

effectively reduce foodborne pathogens, nevertheless, their use may be hindered due to the complex nature of poultry skin.

Although SBS proved to be a potent antimicrobial in the current study, there is a possible buffering effect of poultry meat and skin that may inhibit the competency of antimicrobials on poultry parts. Tan et al. (31) demonstrated that the use of organic acids was capable of reducing Salmonella on chicken skin. However, the use of organic acids was only effective after the $\mathrm{pH}$ was reduced below 2, with acetic acid being the most efficacious (31). This is consistent as chicken skin exhibits a stronger buffering effect than skin remnants and adipose tissue alone (32). As a result, the efficacy of SBS may be inhibited, but the application of a surfactant in conjunction with SBS may counteract some of the potential buffering ability of poultry skin and meat. This can potentially be overcome with the use of surfactants, which disrupt the surface topography of the skin and reduce the buffering effect. To illustrate the advantage of combining inorganic acids with surfactants, Kim and Day (33) combined hydrogen peroxide, sodium bisulfate, and thymol, a surfactant, and evaluated the effect of the combined solutions on E. coli and S. Typhimurium. Kim and Day (33) demonstrated the combination yielded a synergistic effect on MIC's as the combination lowered E. coli and S. Typhimurium three-fold greater than the MIC's of the individual components and reduced both pathogens by 2 logs. As a result, the incorporation of a surfactant such as thymol should be included in future studies to enhance the anti-pathogenic effects of SBS and PAA.

Another factor that may have also played a role in the inhibition of SBS was the acquisition of drumsticks from a local supermarket rather than acquiring them immediately after cut-up in a local processing plant. Therefore, the drumsticks purchased may have had antimicrobials such as PAA already applied to them. Although this may have influenced the results, the effect in the current study would be relatively small as untreated controls were utilized. Thus, any bias toward pretreatment of drumsticks should be accounted for when comparing the results. In addition, because of the small sample size ( $n=4$ per treatment) used in the current experiment, there is a room for future research to validate our current result with a larger sample size.

\section{CONCLUSIONS}

The current study demonstrated that there is a greater efficacy on $S$. Enteritidis reduction as SBS concentration is increased, with no visual discoloration and 3\% SBS being most effective. Drumsticks treated with 3\% SBS, 2\% SBS with the addition of $200 \mathrm{ppm}$ of PAA, and 3\% SBS with the addition of $200 \mathrm{ppm}$ of PAA had the most significant reductions of $S$. Enteritidis over a 3 -d refrigeration period (1.7 log CFU of $S$. Enteritidis per $g$ of drumstick of $S$. Enteritidis). The treatment of drumsticks with $3 \%$ SBS demonstrated the effective reduction of $S$. Enteritidis regardless of the presence of $200 \mathrm{ppm}$ of PAA. Therefore, the application of $3 \%$ SBS as an antimicrobial part dip has the potential to be an advantageous tool to further reduce the contamination of poultry parts past the post-chilling stages of processing.

Further research should be conducted to determine the effects these specific concentrations of SBS have on the overall shelf life of poultry parts and on diminishing Salmonella when combined with a surfactant. In order to determine whether or not efficacy is consistent across all major poultry serovars, SBS needs to be tested with other Salmonella serovars. Lastly, studies that optimize the application of SBS to reduce Salmonella and determine other potentially synergistic compounds must be conducted. In doing so, investigators will continue to develop potent antimicrobials for poultry processing that will reduce the transmission of pathogens to the food supply.

\section{AUTHOR CONTRIBUTIONS}

All authors significantly contributed to the work of the current study. DD, JA, KF, and SR designed and prepared the current study with the assistance from CK. DD and JA conducted the experiment. JL analyzed the data and DD wrote the manuscript with assistance from KF, JL, CK, and SR.

\section{ACKNOWLEDGMENTS}

The authors thank Laura R. Meyer for her technical assistance on this project. The author DD would like to acknowledge the Graduate College at the University of Arkansas for its support through the Distinguished Academy Fellowship and the continued support from the Center for Advanced Surface Engineering, under the National Science Foundation Grant No. OIA-1457888 and the Arkansas EPSCoR Program, ASSET III. Finally, the authors would like to thank Jones-Hamilton Co. for its support and assistance in completing this research. The Foundation for Meat \& Poultry Research \& Education is acknowledged for its support. 


\section{REFERENCES}

1. Hulebak KL, Schlosser W. Hazzard analysis and critical control point (HAACP) history and conceptual overview. Risk Anal. (2002) 22:547-52. doi: $10.1111 / 0272-4332.00038$

2. FDA. Public Law 111-353 in - 111th Congress: FDA Food Safety Modernization Act. Washington DC (2011).

3. Finstad S, O'Bryan C, Marcy JA, Crandall PG, Ricke SC. Salmonella and broiler production in the United States: relationship to foodborne salmonellosis. Food Res Int. (2012) 45:789-94. doi: 10.1016/j.foodres.2011.03.057

4. Ricke SC. Insights and challenges of Salmonella infections in laying hens. Curr Opin Food Sci. (2017) 18:43-9. doi: 10.1016/j.cofs.2017.10.012

5. Food and Drug Administration-Center for Food Safety and Applied Nutrition (FDA-CFSAN). Produce safety from production to consumption: 2004 action plan to minimize foodborne illness associated with fresh produce consumption. Federal Reg. (2004) 69:33393-4.

6. McKee S. "Salmonella control in poultry processing," In: Proceedings of the American Meat Science Association $65^{\text {th }}$ Annual Reciprocal Meat Conference. Fargo, SD: North Dakota State University Fargo (2012).

7. Dincer AH, Baysal T. Decontamination techniques of pathogen bacteria in meat and poultry. Crit Rev Microbiol. (2004) 30:197-204. doi: 10.1080/10408410490468803

8. Loretz M, Stephan R, Zweifel C. Antimicrobial activity of decontamination treatments for poultry carcasses: a literature survey. Food Contr. (2010) 21:791-804. doi: 10.1016/j.foodcont.2009.11.007

9. Shue CW, Freese E. Lipopolysaccharide layer protection of Gram-negative bacteria against inhibition by long-chain fatty acids. J Bacteriol. (1973) 115:869-75.

10. Ricke SC. Perspectives on the use of organic acids and short chain fatty acids as antimicrobials. Poult Sci. (2003) 82:632-9. doi: 10.1093/ps/82.4.632

11. Foster JW, Hall HK. Inducible $\mathrm{pH}$ homeostasis and the acid tolerance response of Salmonella typhimurium, J Bacteriol. (1991) 173:5129-35. doi: 10.1128/jb.173.16.5129-5135.1991

12. Foster JW. The acid tolerance response of Salmonella typhimurium involves the synthesis of key acid shock proteins. J Bacteriol. (1993) 75:1981-7. doi: 10.1128/jb.175.7.1981-1987.1993

13. Foster JW. Microbial Responses to Acid Stress. Bacterial Stress Responses, Chapter. 7. Washington, DC: ASM Press (2000). p. 99-115.

14. Bearson S, Bearson B, Foster J. Acid stress responses in enterobacteria. FEMS Microbiol Lett. (1997) 147:173-80. doi: 10.1111/j.1574-6968.1997.tb1 0238.x

15. Knueven CJ. Sodium Bisulfate as Acidulant in Foods. U.S. Patent No 5,958,491. Washington, DC: Patent US, and Trademark Office (1999).

16. Hill C, O'Driscoll B, Booth I. Acid adaption and food poisoning microorganisms. Int J Food Microbiol. (1995) 28:245-54.

17. Payne JB, Kroger EC, Watkins SE. Evaluation of litter treatments on Salmonella recovery from poultry litter. J Appl Poult Res. (2002) 11:239-43. doi: 10.1093/japr/11.3.239

18. Ruiz-Feria CA, Larrison E, Davis M, Farnell M, Carey J, Grimes JL, et al. Supplementation of feed grade sodium bisulfate in broiler diets improves feed efficiency Int J Poult Sci. (2011) 10:670-6. doi: 10.3923/ijps.2011. 670.676

19. Kim SA, Park SH, Knueven C, Basel R, Ricke SC. A decontamination approach using a combination of bisulfate soda and peracetic acid against Listeria innocua inoculated on whole apples. J Food Cont. (2018) 84:106-10. doi: 10.1016/j.foodcont.2017. 07.036
20. United States Environmental Protection Agency (EPA). Safer Chemical Ingredients List. (2018). Available online at: https://www.epa.gov/saferchoice/ safer-ingredients (Accessed September 25, 2018).

21. World Health Organization. Compendium of Food Additive Specifications. FAO JECFA Monographs 4. (2007). Available online at: http://www.fao.org/ 3/a-a1447e.pdf (Accessed December 6, 2018).

22. USDA FSIS. FSIS Notice 41-16. Washington DC (2016) (Accessed June 8, 2016).

23. R Core Team. R: A Language and Environment for Statistical Computing. Vienna: R Foundation for Statistical Computing (2018). Available online at: http://www.R-project.org/

24. Chen HM, Wang Y, Su LH, Chu CH. Nontyphoid Salmonella infection: microbiology, clinical features and antimicrobial therapy. Pediatr. Neonatal. (2013) 54:147-52. doi: 10.1016/j.pedneo.2013.01.010

25. McEntire J, Acheson D, Siemens A, Eilert S, and Robach M. The public health value of reducing Salmonella levels in raw meat and poultry. Food Protect Trends (2014) 34:386-92.

26. Brinez WJ, Roig-Sagues AX, Hernandez Herrero MM, Lopez-Pedemonte T, Guamis B. Bactericidal efficacy of peracetic acid in combination with hydrogen peroxide against pathogenic and non pathogenic strains of Staphylococcus spp., Listeria spp., and Escherichia coli. Food Cont. (2006) 17:516-21. doi: 10.1016/j.foodcont.2005.02.014

27. Bauermeister LJ, J.Bowers WJ, Townsend JC, McKee SR. Validating the efficacy of peracetic acid mixture as an antimicrobial in poultry chillers. J. Food Protect. (2008) 71:1119-22. doi: 10.4315/0362-028X-71.6.1119

28. Nagel GM, Bauermeister LJ, Bratcher CL, Singh M, McKee SR. Salmonella and Campylobacter reduction and quality characteristics of poultry carcasses treated with various antimicrobials in a post-chill immersion tank. Int. J. Food Microbiol. (2013) 165:281-6. doi: 10.1016/j.ijfoodmicro.2013.05.016

29. Li Y, Slavik MF, Walker JT, Xiong H. Pre-chill spray of chicken carcasses to reduce Salmonella Typhimurium. J Food Sci. (1997) 62:605-7. doi: 10.1111/j.1365-2621.1997.tb04441.x

30. Yang Z, Li Y, Slavik M. Use of antimicrobial spray applied with an insideoutside birdwasher to reduce bacterial contamination on prechilled chicken carcasses. J Food Prot. (1998) 61:829-32. doi: 10.4315/0362-028X-61.7.829

31. Tan SM, Lee SM, Dykes GA. Buffering effect of chicken skin and meat protects Salmonella enterica strains against hydrochloric acid but not organic acid treatment. J Food Cont. (2014) 42:329-34. doi: 10.1016/j.foodcont.2014.02.031

32. Tan SM, Lee SM, Dykes GA. Fat contributes to the buffering capacity of chicken skin and meat but enhances the vulnerability of attached Salmonella cells to acetic acid treatment. Food Res Int. (2014) 66:417-23. doi: 10.1016/j.foodres.2014.10.007

33. Kim D, Day DF. A biocidal combination capable of sanitizing raw chicken skin. Food Contr. (2007) 18:1272-6. doi: 10.1016/j.foodcont.2006.08.004

Conflict of Interest Statement: CK is employed by the company Jones-Hamilton.

The remaining authors declare that the research was conducted in the absence of any commercial or financial relationships that could be construed as a potential conflict of interest.

Copyright (c) 2019 Dittoe, Atchley, Feye, Lee, Knueven and Ricke. This is an openaccess article distributed under the terms of the Creative Commons Attribution License (CC BY). The use, distribution or reproduction in other forums is permitted, provided the original author(s) and the copyright owner(s) are credited and that the original publication in this journal is cited, in accordance with accepted academic practice. No use, distribution or reproduction is permitted which does not comply with these terms. 